\title{
Association of Anti-Ro/Sjögren's syndrome type A Antibodies and Complete Atrioventricular Block in an Adult With Sjögren's Syndrome
}

\author{
Weng Kin TAM, ${ }^{1}$ Hui Ching HSU, ${ }^{2,4}$ Ming Hsiung HSIEH, ${ }^{3,4}$ Jong Shiuan YEH, ${ }^{3,4}$ Weng Chio TAM ${ }^{3,4}$ \\ ${ }^{1}$ Department of Psychiatry, Wan Fang Hospital, Taipei Medical University, Taipei, Taiwan \\ ${ }^{2}$ Department of Internal Medicine, Division of Rheumatology, Wan Fang Hospital, Taipei Medical University, Taipei, Taiwan \\ ${ }^{3}$ Department of Internal Medicine, Division of Cardiovascular Medicine, Wan Fang Hospital, Taipei Medical University, Taipei, Taiwan \\ ${ }^{4}$ Department of Internal Medicine, School of Medicine, College of Medicine, Taipei Medical University, Taipei, Taiwan
}

\begin{abstract}
A 57-year-old female patient with underlying hypertension, dyslipidemia, membranous glomerulonephritis, and Sjögren's syndrome (SS) presented to our clinic with near-syncope and dizziness symptoms. We detected non-conducted sinus beat in 12-lead electrocardiography. An echocardiography showed that the left ventricular systolic function was preserved without a regional wall abnormality. The patient did not have symptoms of congestive heart failure or recent myocardial infarction. We observed complete atrioventricular block (CAVB) with longest pause of 6.2 seconds in a 24-hour Holter electrocardiography, and subsequently implanted a permanent pacemaker. Anti-Ro/SS type A antibodies are commonly seen in SS and associated with a neonatal complete heart block. The pathogenesis of anti-Ro/SS type $A$ antibodies in the conduction system of an adult heart is still uncertain. In this article, we report SS in an adult patient with CAVB and membranous glomerulonephritis, which may be associated with a positive titer of anti-Ro/SS type A antibodies.

Keywords: Anti-Ro/Sjögren's syndrome type A antibodies; complete atrioventricular block; Sjögren's syndrome.
\end{abstract}

The development of complete heart block $(\mathrm{CHB})$ is a major manifestation of cardiac involvement within neonatal lupus syndrome. It is a congenital disorder and associated with the placental transmission of maternal anti-Ro/Sjögren's syndrome type A (anti-Ro/SSA) antibodies. The neonatal atrioventricular node is vulnerable to the impaired electrical coupling secondary to apoptosis, dysfunctional calcium channels, and fibrosis. ${ }^{1}$ It is still unknown whether this autoimmunity phenomenon is pathogenic to the adult heart. Moreover, renal involvement was previously reported in autoimmune diseases. Distal renal tubular acidosis or mesangioproliferative glomerulonephritis is the most common renal manifestation of Sjögren's syndrome (SS) that is mediated by the pathogenesis of tubulointerstitial nephritis or immune complex-mediated glomerulopathy. ${ }^{2}$
There are few reports of adults with SS along with membranous glomerulonephritis and a $\mathrm{CHB}^{3,4}$ In this case report, we present a patient with anti-Ro/SSA positive SS and membranous glomerulonephritis, which may be associated with cardiac conduction disturbance and a CHB. Thus, we suggest that middle-aged patients with SS and clinical symptoms of dizziness or near-syncope should be alerted about the possibility of insidious heart conduction problems.

\section{CASE REPORT}

A 57-year-old female patient had a medical history of hypertension and dyslipidemia. Five years ago, she had an initial presentation of dry eye and mouth symptoms for a period of six months. Sialoscintigraphy showed impaired function of

Received: April 27, 2017 Accepted: July 17, 2017 Published online: October 12, 2017

Correspondence: Weng Chio Tam, MD. Department of Internal Medicine, Division of Cardiovascular Medicine, Wan Fang Hospital, Taipei Medical University, 116 Taipei, Taiwan. Tel: 886919585771 e-mail: chio_2001_2@hotmail.com

@2018 Turkish League Against Rheumatism. All rights reserved. 

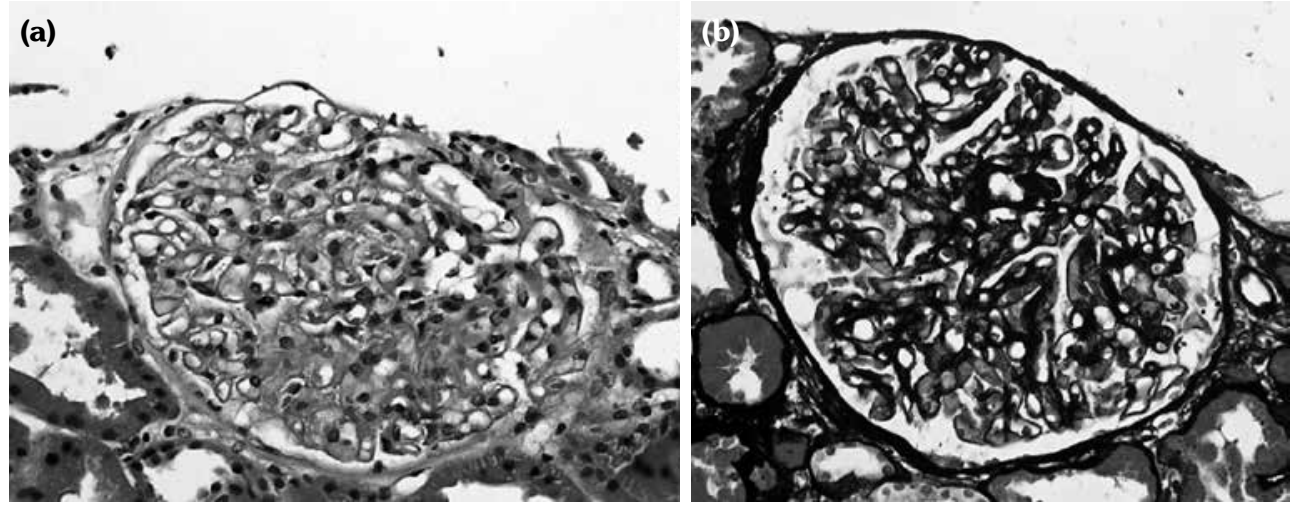

Figure 1. (a) Light microscopic appearance of membranous nephropathy is seen in rounded capillary lumina with mild thickening of glomerular basement membrane in all non-sclerotic glomeruli. Global sclerosis is observed in five out of 11 glomeruli. (b) A silver stain of glomerulus highlighting proteinaceous basement membranes (black) is noted. Hematoxylin \& eosin stain and sliver stain with very high magnification (x400).

the bilateral submandibular and parotid glands. The result of Schirmer's test (right eye) was about $4 \mathrm{~mm}$. Antinuclear antibodies titer was 1:160 with positive speckled pattern. Anti-Ro/SSA was positive $(146.0 \mu / \mathrm{mL}$, normal: $7-10 \mu / \mathrm{mL})$ and Anti-La/Sjögren's syndrome type B was negative. Anti-double stranded deoxyribonucleic acid was not found. There was no musculoskeletal, neurological or vascular involvement. The patient was diagnosed with primary SS and received regular follow-up at outpatient clinics where hydroxychloroquine (200 mg daily) and symptomatic relief was given. Subsequently, the patient developed proteinuria. After a renal biopsy, the patient was diagnosed with membranous glomerulonephritis (Figures 1a, b), apart from tubulointerstitial nephritis or mesangioproliferative glomerulonephritis. Additionally, the patient was given diuretics, angiotensin II receptor blocker, and statin. After medical therapy, the patient's clinical condition improved, and as a result, she did not undergo any immunosuppressive therapy. The patient visited our cardiology outpatient clinic because of her symptoms of dizziness and nearsyncope for several months. Physical examination showed a regular heart beat associated with grade II/VI systolic murmur over the apex. Serum free $\mathrm{T}_{4}$ and creatinine levels were $1.23 \mathrm{ng} / \mathrm{dL}$ (0.7-1.8 ng/dL) and $0.85 \mathrm{mg} / \mathrm{dL}(0.6-1.3 \mathrm{mg} / \mathrm{dL})$, respectively. Serum electrolyte levels, including sodium, potassium, calcium and phosphorous were also within the normal limit. Although chest radiography was borderline positive for cardiomegaly, the patient denied having symptoms of congestive heart failure, such as shortness of breath or exercise intolerance. A 12-lead electrocardiography (ECG) demonstrated a sinus rhythm and one non-conducted sinus beat at the outpatient clinic (Figure 2a). The echocardiography showed a preserved left ventricle systolic function without a regional wall abnormality, but a 24-hour
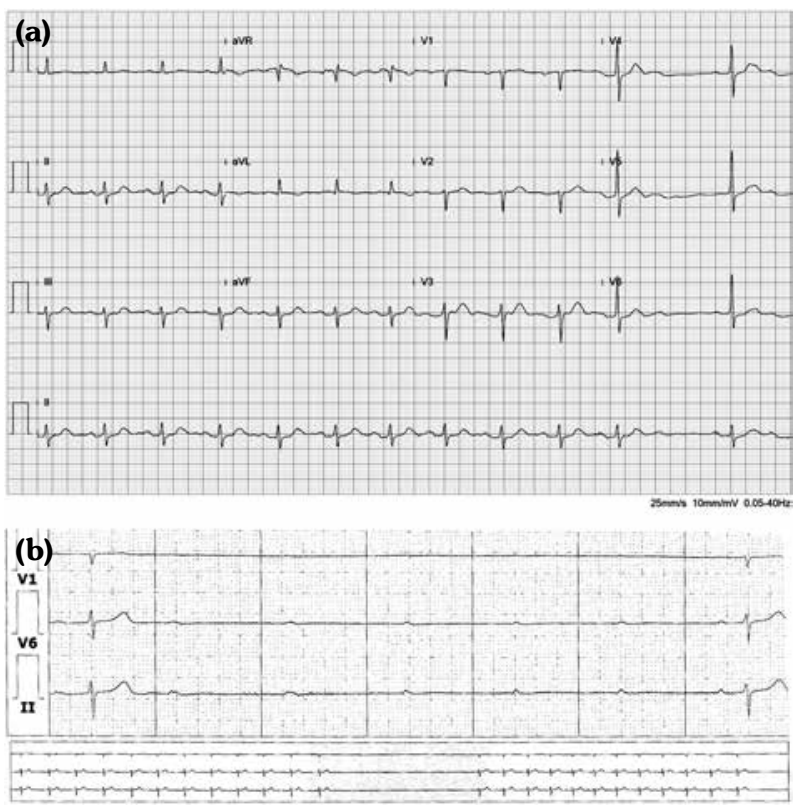

Figure 2. (a) A 12-lead electrocardiography showing non-conducted sinus beat. (b) Complete atrioventricular block with a long pause of 6.2 seconds in 24 hours (Holter electrocardiography). 
Table 1. Association between Anti-Ro/SSA and complete atrioventricular block in patients with autoimmune disease

\begin{tabular}{|c|c|c|c|c|c|}
\hline Authors & Year & Study category & Autoimmune disease & Conduction defect & $\begin{array}{l}\text { Number of adults with } \\
\text { CAVB and positive } \\
\text { Anti-Ro/SSA }\end{array}$ \\
\hline Maier et al. ${ }^{6}$ & 1987 & Case Report & SLE & CAVB & 1 \\
\hline Behan et al. ${ }^{16}$ & 1987 & Case-control & $\mathrm{PM} / \mathrm{DM}$ & CAVB & 4 \\
\hline Mevorach et al. ${ }^{7}$ & 1993 & Case Report & SLE & CAVB & 1 \\
\hline Lee et al. ${ }^{17}$ & 1996 & Case Report & pSS & CAVB & 1 \\
\hline Comin-Colet et al. ${ }^{8}$ & 2001 & Case Report & SLE & CAVB & 1 \\
\hline Lim and Joshua9 & 2005 & Case Report & SLE & CAVB & 1 \\
\hline Arce-Salinas et al. ${ }^{10}$ & 2009 & Case Report & SLE & CAVB & 1 \\
\hline Sung et $\mathrm{al}^{3}$ & 2011 & Case report & pSS & CAVB & 1 \\
\hline
\end{tabular}

Holter ECG exhibited episodes of complete atrioventricular blocks (CAVB) with a long pause of 6.2 seconds (Figure 2b). The patient was hospitalized and eventually received permanent pacemaker implantation, after which the patient's symptoms of dizziness and general weakness improved completely. A written informed consent was obtained from the patient.

\section{DISCUSSION}

Transplacental passage of anti-Ro/SSA antibodies is a well-documented cause of atrioventricular block and conduction defects in newborns with neonatal lupus syndrome. However, this is rarely reported in adults. In this case study of a middle-aged patient with positive anti-Ro/SSA antibodies and CAVB, we suggest that the antibodies may be critically involved in the development of conduction disturbances in certain adults. The potential pathophysiology and mechanism may be partially or completely different from the conduction defects found in neonatal lupus syndrome. We speculate that anti-Ro/SSA antibodies may be arrhythmogenic for adults.

Although anti-Ro/SSA antibodies are conclusively associated with neonatal $\mathrm{CHB}$, some newborns with the existence of anti-Ro/SSA antibodies and neonatal lupus syndrome may develop heart block in adulthood. ${ }^{5}$ Moreover, adult patients with systemic lupus erythematous and anti-Ro/SSA antibodies have been reported to have a transient or $\mathrm{CHB} .{ }^{6-10}$ It remains unclear whether the adult heart is resistant or vulnerable to anti-Ro/SSA. To the best of our knowledge, the theories of calcium channelopathy, electrophysiological effects of myocardium, and inflammation have been attributed to the pathogenesis of anti-Ro/SSA. ${ }^{11}$

For the theory of calcium channelopathy, Lazzerini et al. ${ }^{12}$ demonstrated neonatal heart involvement and arrhythmogenic impact on an adult heart using anti-Ro/SSA antibodies. Anti-Ro/SSA antibodies could be related to the dysregulation of calcium channels, as highly expressed in the atrioventricular and sinoatrial nodes. The conduction system tissue damage may be related to higher calcium channel reserves and lower intrinsic sensitivity of adult cardiomyocytes. Calcification and fibrotic tissue are deposited at the atrioventricular node and other conduction systems. Fibroblasts can also significantly reduce electrical coupling, while also slowing down and blocking the conduction system, which may be a cause of $\mathrm{CHB}$ in adult patients.

The electrophysiological effect of myocardium from anti-Ro/SSA antibodies in adults has also been documented. Anti-Ro/SSA antibodies can induce cardiac conduction abnormalities in the hearts of adult rabbits. ${ }^{13}$ However, the characteristics of neonatal human atrial cells are different from adult cells. Wang et al. ${ }^{14}$ found larger current density, faster inactivation, and slower recovery from inactivation in the neonatal atrial cells compared with that of the adults. This may explain why the neonatal atrial cells are vulnerable to a conduction delay after an anti-Ro/SSA antibody attack. In vitro research has demonstrated that the interaction of anti-Ro/SSA antibodies with potassium -interaction between the cardiac 
rapidly- channel and human ether-a-go-go related gene-channel protein develop arrhythmogenicity in adults with torsades de pointes. It was also reported that anti-Ro/SSA antibodies may interfere with the electrophysiological properties of ventricular repolarization. ${ }^{15}$

Previous studies have demonstrated that anti-Ro/SSA antibodies are associated with the incidence of a CAVB, mostly in patients with systemic lupus erythematosus, but rarely in patients with primary SS (Table 1). $3,6-8,10,11,16,17$ Although our patient had other possible risk factors, such as aging or hydroxychloroquine cardiotoxicity for a CAVB ${ }^{18}$ anti-Ro/SSA antibodies should also be considered as a potential cause for CAVB. However, additional studies with larger sample sizes should be carried out to warrant this result.

Heart block in adults with SS is classified into acquired and late progressive congenital forms. ${ }^{11}$ Regardless of the prevalence of an autoimmune disease, the absence of anti-Ro/SSA antibodies in the patient's mother is required to diagnose the acquired form. In the acquired form, the conduction defect may be related to the calcium channel and electrophysiological interference of anti-Ro/SSA antibodies, and as a result, immunosuppressive agents may be more effective in recovering the function and integrity of the cardiac conduction system. ${ }^{11,19}$

Sjögren's syndrome is a slowly progressing disease that is different from lupus erythematous and rheumatoid arthritis in its robust clinical manifestation. Other sites of extra-glandular inflammation include the lung, blood vessel, skin, and heart. Alterations of renal function in SS are common. Two forms of renal involvement with primary SS can be found in $4.2-67 \%$ of patients, due to lymphocytic infiltration or an immunecomplex deposition. ${ }^{2}$

In conclusion, we have documented this case report on a patient with SS, membranous glomerulonephritis and an initial presentation of one non-conducted sinus beat in a 12-lead ECG. Eventually, the patient had an occult CAVB, besides SS and extra-glandular involvement. Diagnostic tools, including a 24-hour Holter ECG and electrophysiological study, should be considered in evaluating the insidious CAVB associated with the existence of anti-Ro/ SSA antibodies, particularly in some patients with near-syncope and dizziness symptoms. This case report indicates that anti-Ro/SSA antibodies are also associated with adult-onset of CAVB. Further studies on the pathogenic role of anti-Ro/SSA are necessary to clarify the precise and putative mechanism that involves conduction disturbances. This knowledge may help us to identify better therapeutic options and diagnostic screenings for this specific population.

\section{Declaration of conflicting interests}

The authors declared no conflicts of interest with respect to the authorship and/or publication of this article.

\section{Funding}

This study was supported by 105-wf-eva-15 Wan Fang Hospital Taipei Medical University.

\section{REFERENCES}

1. Santos-Pardo I, Villuendas R, Salvador-Corres I, Martínez-Morillo M, Olivé A, Bayes-Genis A. AntiRo/SSA antibodies and cardiac rhythm disturbances: Present and future perspectives. Int $\mathrm{J}$ Cardiol 2015;184:244-50.

2. Kronbichler A, Mayer G. Renal involvement in autoimmune connective tissue diseases. BMC Med 2013;11:95.

3. Sung MJ, Park SH, Kim SK, Lee YS, Park CY, Choe JY. Complete atrioventricular block in adult Sjögren's syndrome with anti-Ro autoantibody. Korean J Intern Med 2011;26:213-5.

4. Baba A, Hara S, Sato Y, Yamada K, Fujimoto S, Eto $\mathrm{T}$. Three patients with nephrotic syndrome due to membranous nephropathy complicated by Sjögren's syndrome. Nihon Jinzo Gakkai Shi 2005; 47:882-6.

5. Capone C, Buyon JP, Friedman DM, Frishman WH. Cardiac manifestations of neonatal lupus: a review of autoantibody-associated congenital heart block and its impact in an adult population. Cardiol Rev 2012;20:72-6.

6. Maier WP, Ramirez HE, Miller SB. Complete heart block as the initial manifestation of systemic lupus erythematosus. Arch Intern Med 1987;147:170-1.

7. Mevorach D, Raz E, Shalev O, Steiner I, BenChetrit E. Complete heart block and seizures in an adult with systemic lupus erythematosus. A possible pathophysiologic role for anti-SS-A/Ro and anti-SS-B/ La autoantibodies. Arthritis Rheum 1993;36:259-62.

8. Comin-Colet J, Sanchez-Corral MA, Alegre-Sancho $\mathrm{JJ}$, Valverde J, Lopez-Gomez D, Sabate X, et al. Complete heart block in an adult with systemic lupus 
erythematosus and recent onset of hydroxychloroquine therapy. Lupus 2001;10:59-62.

9. Lim LT, Joshua F. Resolution of complete heart block after prednisolone in a patient with systemic lupus erythematosus. Lupus 2005; 14:561-3.

10. Arce-Salinas CA, Carmona-Escamilla MA, RodríguezGarcía F. Complete atrioventricular block as initial manifestation of systemic lupus erythematosus. Clin Exp Rheumatol 2009;27:344-6.

11. Lazzerini PE, Capecchi PL, Laghi-Pasini F. Isolated atrioventricular block of unknown origin in adults and anti-Ro/SSA antibodies: clinical evidence, putative mechanisms, and therapeutic implications. Heart Rhythm 2015;12:449-54.

12. Lazzerini PE, Capecchi PL, Laghi-Pasini F. AntiRo/SSA antibodies and cardiac arrhythmias in the adult: facts and hypotheses. Scand $\mathrm{J}$ Immunol 2010;72:213-22.

13. Garcia S, Nascimento JH, Bonfa E, Levy R, Oliveira $\mathrm{SF}$, Tavares AV, et al. Cellular mechanism of the conduction abnormalities induced by serum from anti-Ro/SSA-positive patients in rabbit hearts. J Clin
Invest 1994;93:718-24.

14. Wang Y, Xu H, Kumar R, Tipparaju SM, Wagner MB, Joyner RW. Differences in transient outward current properties between neonatal and adult human atrial myocytes. J Mol Cell Cardiol 2003;35:1083-92.

15. Lazzerini PE, Yue Y, Srivastava U, Fabris F, Capecchi PL, Bertolozzi I, et al. Arrhythmogenicity of Anti-Ro/ SSA Antibodies in Patients With Torsades de Pointes. Circ Arrhythm Electrophysiol 2016;9:3419.

16. Behan WM, Behan PO, Gairns J. Cardiac damage in polymyositis associated with antibodies to tissue ribonucleoproteins. Br Heart J 1987;57:176-80.

17. Lee LA, Pickrell MB, Reichlin M. Development of complete heart block in an adult patient with Sjögren's syndrome and anti-Ro/SS-A autoantibodies. Arthritis Rheum 1996;39:1427-9.

18. Tönnesmann E, Kandolf R, Lewalter T. Chloroquine cardiomyopathy - a review of the literature. Immunopharmacol Immunotoxicol 2013;35:434-42.

19. Santos-Pardo I, Martínez-Morillo M, Villuendas R, Bayes-Genis A. Anti-Ro antibodies and reversible atrioventricular block. N Engl J Med 2013;368:2335-7. 